\title{
COMPUTATIONS ON THE INTERACTION OF AN \\ INTERPLANETARY SHOCK WITH THE EARTH'S \\ MA GNETOSPHERE
}

\author{
(Letter to the Editor)
}

IMKE DE PATER

Sterrewacht, Leiden, The Netherlands

and

WIM J. WEBER

Sterrewacht, Utrecht, The Netherlands

(Received 13 April, 1977)

\begin{abstract}
We present the results of a one-dimensional computer simulation of the interaction between interplanetary shocks and the Earth's magnetosphere. The position of the bowshock as a function of solar wind velocity and interplanetary field direction is studied.
\end{abstract}

\section{Observations}

Rocket and satellite observations show the radial compression of the terrestrial bowshock and magnetopause due to temporary increase of the solar wind density and velocity (Kovalevsky, 1971). Moreover, the Earth-magnetopause distance is shorter in the case when the interplanetary field $B_{\mathrm{ip}}$ is antiparallel to the Earth's field (Aubry et al., 1970).

From the literature we chose HEOS observations of 1969, Febr. 27th (Bonetti et al., 1970; Hedgecock, 1970), when an interplanetary shock hits the bowshock during the observations. Under the assumption that the bowshock and magnetopause are flat, the Rankine-Hugoniot jump equations (Spreiter et al., 1966) can be fulfilled with the quantities presented in the first three columns of Table I. These quantities are in good agreement with the observed solar wind values, while the discrepancy in the sheath due to curvature of the bowshock is small. The stand-off distance of the bowshock $(D+\Delta)$ decreases from $13.6 R_{\mathrm{e}}$ to $11.5 R_{\mathrm{e}}$ along the line Sun-Earth (computed with the aid of the bowshock form derived by Fairfield (1971). The Earth-magnetopause distance $D$, along the same line, was derived from the empirical formula of Spreiter et al. (1966) as

$$
\Delta / D=1.1 \varrho_{0} / \varrho_{1}
$$

which yields $D=10.2 R_{\mathrm{e}}$ during the first bowshock crossing, using $\varrho_{1} / \varrho_{0}=3.32$ as in Table I. 


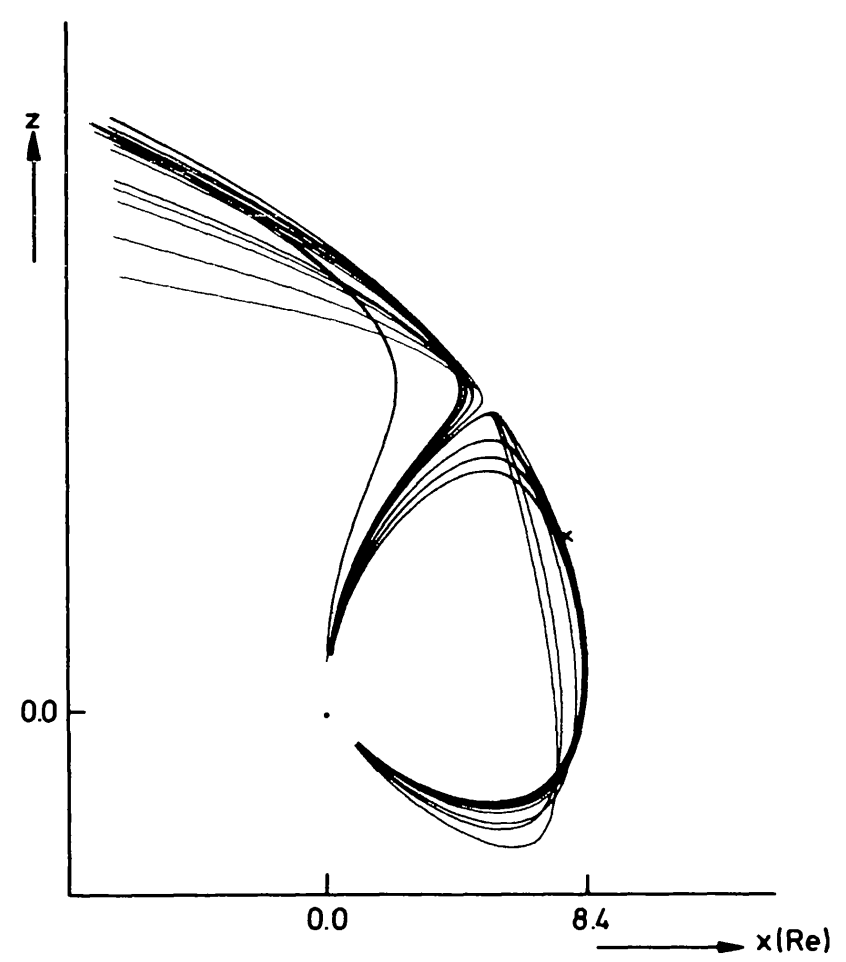

(a)

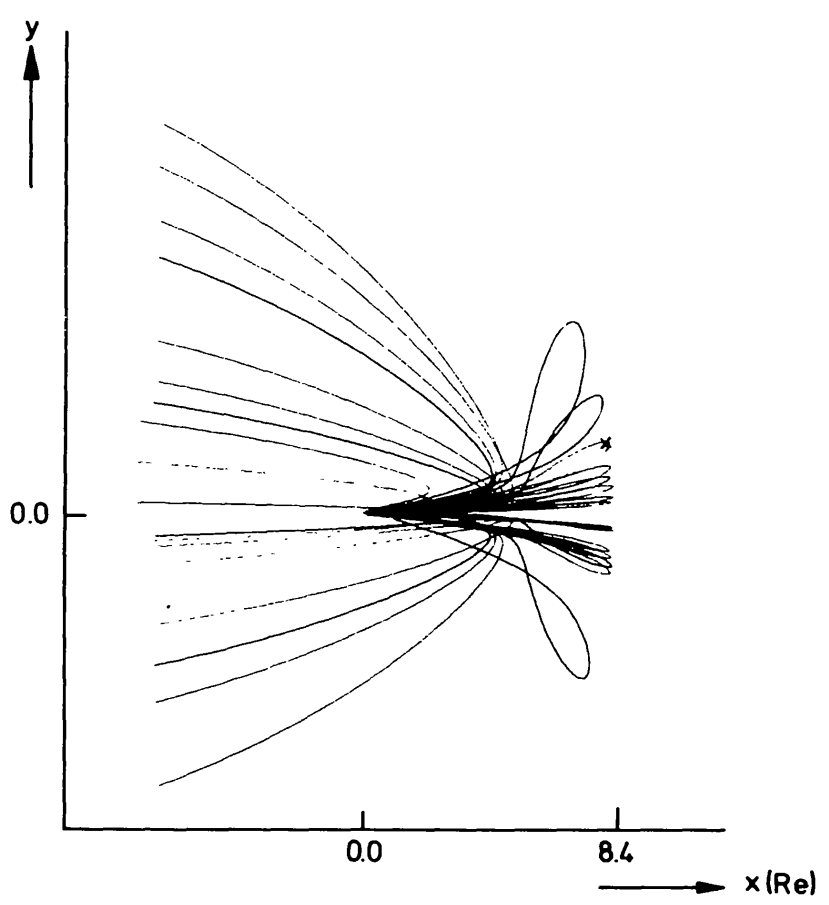

(b)

Figs. 1a-b. The shape of the magnetosphere at noon-side on 1969, Febr. 27th at 0700 UT, with a distance $D=8.4 R_{\mathrm{e}}$. The crosses $\mathrm{x}$ indicate the projection on the $x-z$ and $x-y$ plane of the satellite entering the magnetosphere. 
TABLE I

Solar wind and magnetosheath quantities ${ }^{\mathrm{a}}$

\begin{tabular}{|c|c|c|c|}
\hline $\begin{array}{l}\text { Quiet solar wind } \\
\text { (model) }\end{array}$ & $\begin{array}{l}\text { Disturbed solar } \\
\text { wind (model) }\end{array}$ & $\begin{array}{l}\text { Magnetosheath } \\
\text { (model) }\end{array}$ & $\begin{array}{l}\text { Magnetosheath } \\
\text { (observed) }\end{array}$ \\
\hline$\varrho_{0}=4.5 \mathrm{pr} \mathrm{cm}^{-3}$ & $\varrho_{4}=8 \mathrm{pr} \mathrm{cm}^{-3}$ & $\varrho_{1}=15 \mathrm{pr} \mathrm{cm}^{-3}$ & $\varrho: 25-30 \mathrm{pr} \mathrm{cm}^{-3}$ \\
\hline$v_{0}=440 \mathrm{~km} \mathrm{~s}^{-1}$ & $v_{4}=512 \mathrm{~km} \mathrm{~s}^{-1}$ & $v_{1}=132 \mathrm{~km} \mathrm{~s}^{-1}$ & $v: 170-210 \mathrm{~km} \mathrm{~s}^{-1}$ \\
\hline$B_{0}=8.45 \gamma$ & $B_{4}=15 \gamma$ & $B_{1}=28 \gamma$ & $B: 38-44 \gamma$ \\
\hline$T_{0}=10^{5} \mathrm{~K}$ & $T_{4}=1.8 \times 10^{5} \mathrm{~K}$ & $T_{1}=1.8 \times 10^{6} \mathrm{~K}$ & $T: 2.1-4.9 \times 10^{6} \mathrm{~K}$ \\
\hline
\end{tabular}

a In the first three columns the computed solar wind and magnetosheath quantities are shown; the fourth column gives the observed sheath values after the region was overtaken by the interplanetary shock. The vertical component of the interplanetary field was directed southward during the observations.

The shape of the magnetosphere at given data, under quiet conditions, is given by Figures $1 \mathrm{a}$ and $1 \mathrm{~b}$, which was made with a field-line tracing programme according to Barish (1972). The programme uses an image dipole to represent the interaction of the solar wind and the Earth's magnetic field. The position of the magnetopause can be found from the last closed field lines. From the projection on the $x-z$ and $x-y$ planes of the satellite entering the magnetosphere, the distance $D$ appeared to be $8.4 R_{\mathrm{e}}$. With the distance $D+\Delta=11.5 R_{\mathrm{e}}$, Equation (1) yields a number density in the sheath of $26.8 \mathrm{pr} \mathrm{cm}^{-3}$ at that time.

\section{Model}

We solved the one-dimensional MHD equations including magnetic diffusion by means of the numerical scheme SHASTA/FCT1 (Boris and Book, 1973). To mimic more-dimensionality, we made the Earth-side boundary reflecting to represent the anchoring of field-line tensions, which would be zero in a truly one-dimensional case. The required increase of field strength within the magnetosphere is then eliminated.

To obtain the stationary situation of a bowshock fulfilling the Rankine-Hugoniot equations and a flat tangential-discontinuity at rest representing the magnetopause, we had to artificially reduce the flow to a zero level going through the bowshock towards the magnetopause. This was accomplished by multiplying the flow speed by a function decreasing from one to zero from the bowshock to the magnetopause. This violation of the one-dimensional conservation laws is justified in view of the more-dimensionality of the actual equilibrium state.

We studied the interaction of a shock propagating in the solar wind with this equilibrium system. We expect that the compression of the magnetosphere will depend upon the direction of the interplanetary field with respect to the terrestrial field. In the case of an antiparallel field, magnetic diffusion and annihilation are expected according to Petschek (1963); the field-flow pattern for this mechanism is shown in 
Figure 2. We simulated this process one-dimensionally by an artificial decrease in number density within the transition layer according to:

$$
\Delta \varrho=\left(\varrho_{\text {new }}-\varrho_{4}\right)\left(1-e^{-u_{x 0} t}\right)=\left(\varrho_{\text {new }}-\varrho_{4}\right)\left(1-e^{-\vee \eta t}\right),
$$

where $\Delta \varrho$ is the decrease in number density, $\varrho_{\text {new }}$ the density computed just before this leakage is taken into account and $\varrho_{4}$ the density at a great distance (Table I). We used Petschek's annihilation rate $u_{x_{0}}=\sqrt{ } \eta / t$ (with the electrical resistivity $\eta=c^{2} / 4 \pi \sigma$ ) in the exponent. It will be clear that there is no annihilation at all in case of a zero resistivity. From sate!lite observations in the magnetopause, we can infer that there exists a finite electrical conductivity there (Burlaga and Scudder, 1974) and thus, indeed, annihilation of field can be expected.

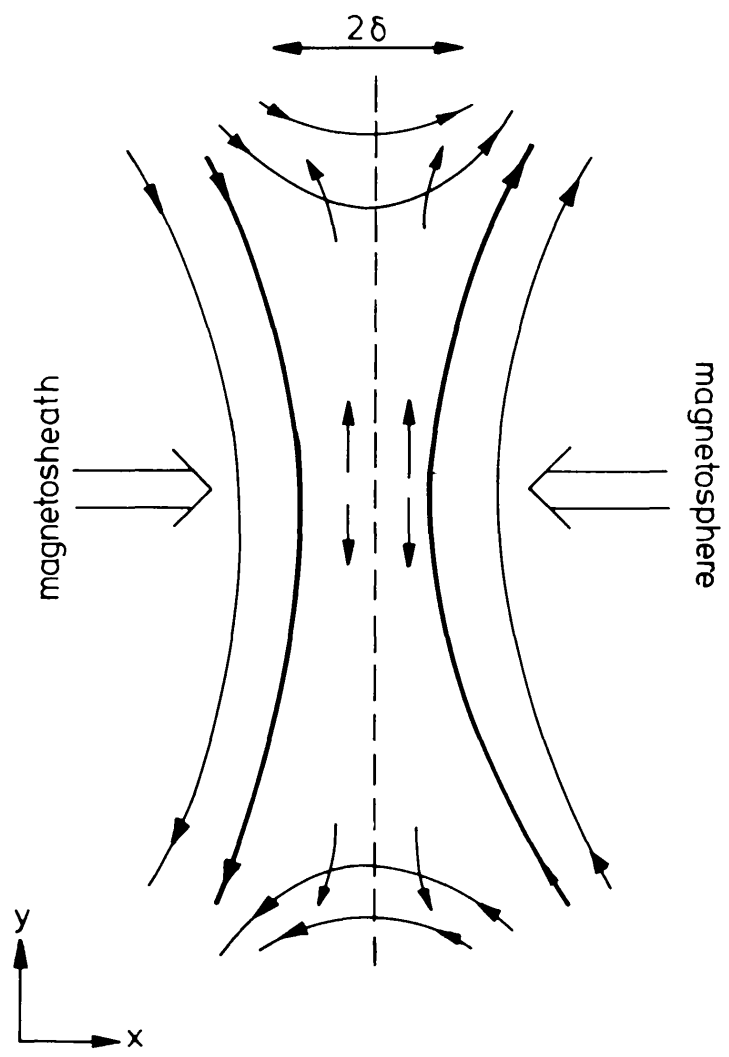

Fig. 2. The reconnection process within the magnetopause according to Petschek (1963): the field lines are transported by the fluid toward the transition layer where an X-type neutral point is formed; here the field is annihilated and the magnetic energy is dissipated into the fluid, which then streams away tangentially along the magnetopause.

\section{Results}

Figures 3 and 4 show what happens according to our computations when an interplanetary shock hits the magnetosphere; Figure 3 the interplanetary field is 


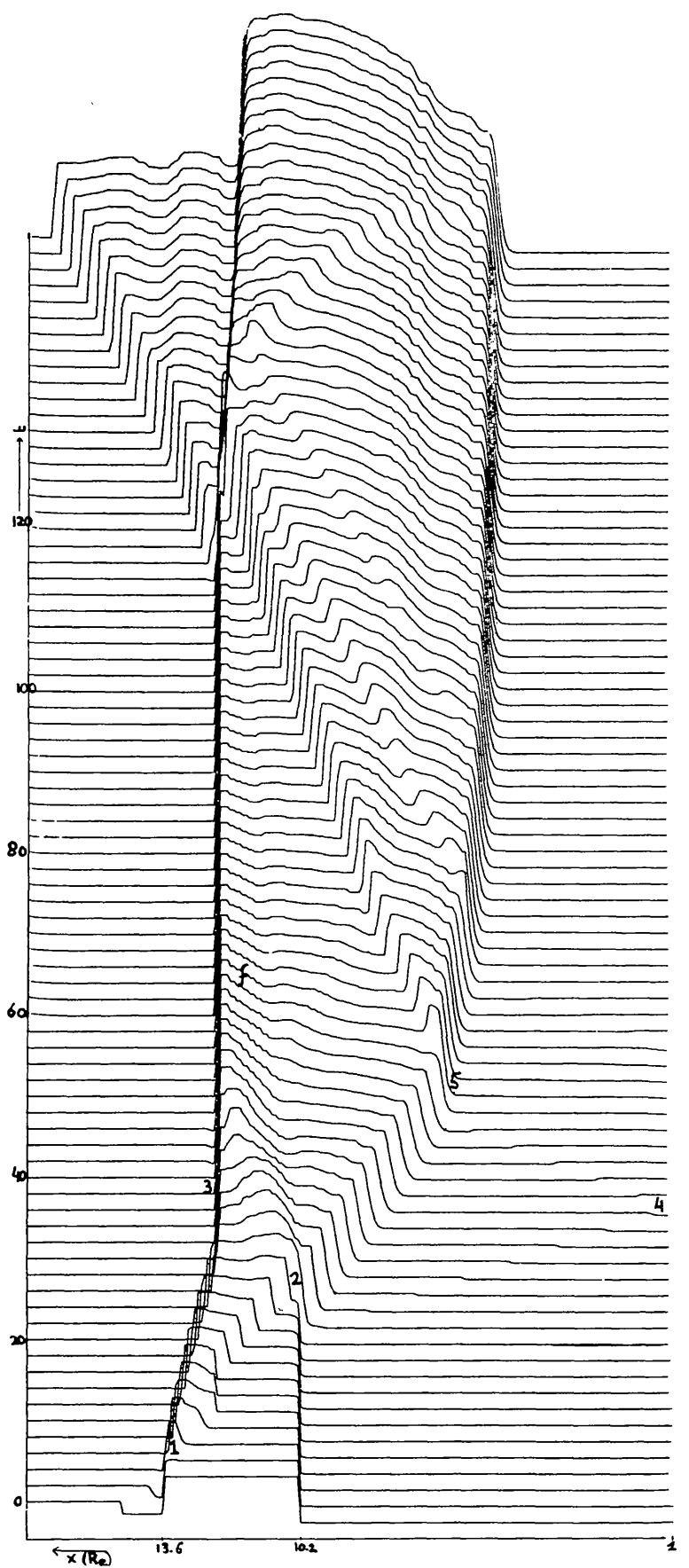

(a)

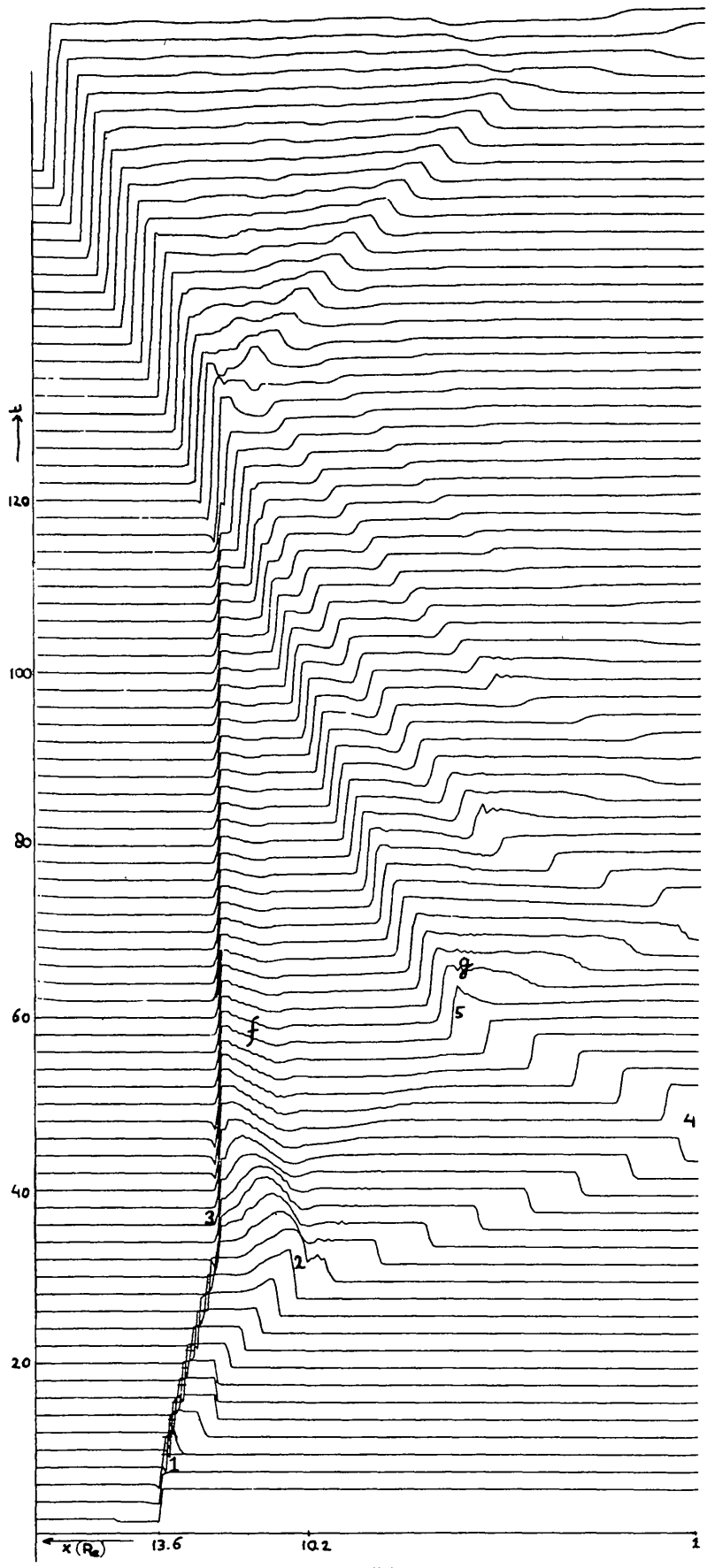

(b)

Fig. 3a. The evolution in density (parallel field situation): the bottom line is drawn at time $t=0$; the $x$ coordinate represents the distance to the Earth in Earth radii $R_{\mathrm{e}}$ and is covered by 200 gridpoints; the $y$ coordinate gives the value of the density relative to the quiet solar wind. After each time interval of 2 time units (which is about 1.8 real seconds) a new line is drawn above the preceding one. See text for further comment.

Fig. 3b. The evolution in total pressure $p+B^{2} / 8 \pi$. 


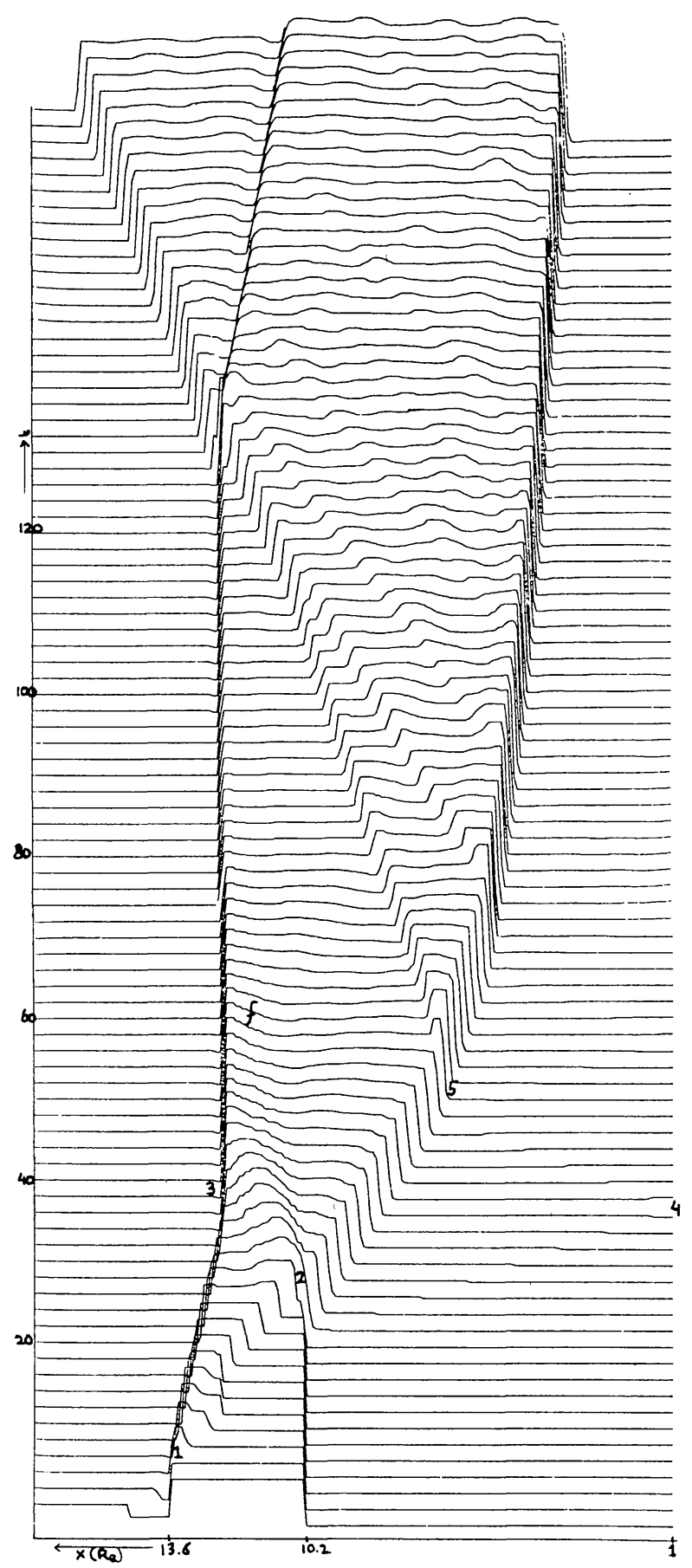

Fig. 4. The evolution in density in an antiparallel field situation. (See caption of Figure 3a.)

parallel to the terrestrial field and in Figure 4 it is antiparallel. In the latter case the annihilation effect was included, with an electrical conductivity of $10^{15} \mathrm{~s}^{-1}$. As a consequence of 'tricks' used in the SHASTA/FCT1 scheme to preserve the monotonicity of initially monotonic distributions, some unrealistic ripples may arise as seen at 
$f$ in the figures; note however that they have no influence on the development of the situation. Neither has the fluttering at $g$ in Figure 3b, which arises from the fact that the total pressure $p+B^{2} / 8 \pi$ is not a directly convected quantity.

One can clearly see the propagation and evolution of the various shocks and waves in the figures: at 1 the interplanetary shock hits the bowshock and two new shocks arise; the shock front propagates toward the magnetopause and hits it at 2; the backend (which now is the bowshock) is moving inward until the wave coming back from 2 stops this motion (at 3). After being hit the magnetopause is pushed toward the Earth and decelerated at 5 by the wave reflected back against the ionosphere at 4 . In Figure $3 \mathrm{~b}$ one can clearly see the zig-zag motion of this shock, with shocks arising each time the magnetopause is hit. Note that they become progressively weaker.

Tables IIa and IIb present some computed physical quantities belonging to Figures 3 and 4, respectively. Keeping in mind the artificial (but not unrealistic) decrease in number density inside the magnetopause, we can understand the differences between the two cases. Note, however, the unrealistic decrease in magnetic field strength as time goes on: this is caused by an acceleration of the particles from the bowshock (where the density is highest) toward the magnetopause, removing the field.

From these and other computations it appears that a variation of a factor 10 in the electrical conductivity greatly influences the development of the antiparallel field situations. Upon comparing the computed quantities at time $\approx 60$ (when moredimensional effects probably will not yet dominate the flow), we conclude that an

TABLE II

Computed magnetosheath quantities ${ }^{\mathrm{a}}$

(a)

\begin{tabular}{rlll}
\hline Time & $\varrho \mathrm{prcm} \mathrm{cm}^{-3}$ & $v \mathrm{~km} \mathrm{~s}^{-1}$ & $B \gamma$ \\
\hline 30 & 30.3 & 163 & 55.4 \\
40 & 25.2 & 193 & 45.4 \\
50 & 28.9 & 199 & 35.0 \\
60 & 30.5 & 188 & 33.3 \\
70 & 31.8 & 181 & 28.6 \\
80 & 32.4 & 178 & 25.1 \\
90 & 32.9 & 177 & 22.4 \\
100 & 52.8 & 68.6 & 33.4 \\
120 & 71.0 & 34.6 & 45.5 \\
\hline
\end{tabular}

(b)

\begin{tabular}{rlll}
\hline Time & $\varrho \mathrm{prcm}^{-3}$ & $v \mathrm{~km} \mathrm{~s}^{-1}$ & $B \gamma$ \\
\hline 24 & 28.2 & 166 & -52.2 \\
36 & 24.4 & 216 & -45.2 \\
48 & 21.6 & 240 & -43.4 \\
60 & 22.0 & 236 & -45.1 \\
72 & 22.0 & 234 & -42.8 \\
84 & 22.0 & 231 & -38.0 \\
96 & 21.6 & 234 & -34.4 \\
108 & 21.7 & 231 & -30.5 \\
120 & 37.3 & 66.8 & -51.9 \\
\hline
\end{tabular}

a The computed physical values in the magnetosheath are shown (in grid 76); Table IIa belongs to Figure 3, Table IIb to Figure 4.

The temperature is about 1 to $1.3 \times 10^{6} \mathrm{~K}$ in both situations, which is a factor of about 3 too low in comparison with the data. The distance $D+\Delta$ is $11.5 R_{\mathrm{e}}$, which is in excellent agreement with the observed distance. The propagation velocity of the magnetopause is, resp.,

$$
\begin{aligned}
& \text { at } t=30: 194 \mathrm{~km} \mathrm{~s}^{-1} 173 \mathrm{~km} \mathrm{~s}^{-1} \\
& \text { at } t=60: \quad 62.7 \mathrm{~km} \mathrm{~s}^{-1} 113 \mathrm{~km} \mathrm{~s}^{-1} \\
& \text { at } t=120: \quad 0.0 \mathrm{~km} \mathrm{~s}^{-1} 20 \mathrm{~km} \mathrm{~s}^{-1}
\end{aligned}
$$


antiparallel field situation with a conductivity of $10^{16} \mathrm{~s}^{-1}$ would fit the observed data reasonably well (disregarding the temperature). At any rate the magnetopause position in this case will be closer to the Earth than in a parallel field situation. In reality, however, the magnetopause would not keep on moving toward the Earth as in the computations, since the region inside the magnetopause, where the field strength is constant (due to magnetopause surface currents), is only 1 to $2 R_{\mathrm{e}}$ thick (Formisano, 1974). Any inward motion of the magnetopause beyond this region will eventually be stopped by the increase in the Earth's dipole-like magnetic field and its associated magnetic pressure.

On the basis of these results we can state that it certainly will be useful to repeat this kind of computation with a two-dimensional programme.

\section{References}

Aubry, M. P., Russel, C. T., and Kivelson, M. G.: 1970, J. Geophys. Res. 75, 7018.

Barish, F.: 1972, Thesis, University of Denver.

Bonetti, A., Moreno, G., Candidi, M., Egidi, A., Formisano, V., and Pizzella, G.: 1970, 'Observation of Solar Wind Discontinuities from February 24 to February 28, 1969', in Intercorrelated Satellite Observations Related to Solar Events (ed. by V. Manno and D. E. Page), D. Reidel Publ. Co., Dordrecht, Holland, p. 436.

Boris, J. P. and Book, D. L.: 1973, J. Computational Phys. 11, 38.

Burlaga, L. F. and Scudder, J. D.: 1974, Astrophys. J. 191, L152.

Fairfield, D. H.: 1971, J. Geophys. Res. 76, 6700.

Formisano, V.: 1974, 'The Earth's Bowshock Fine Structure', in Correlated Interplanetary and Magnetospheric Observations (ed. D. E. Page), D. Reidel Publ. Co., Dordrecht, Holland, p. 187.

Hedgecock, P. C.: 1970, 'The Solar Particle Event of February 25, 1969', in Intercorrelated Satellite Observations Related to Solar Events (ed. by V. Manno and D. E. Page), D. Reidel Publ. Co., Dordrecht, Holland, p. 419.

Kovalevsky, J. V.: 1971, Space Sci. Rev. 12, 187.

Petschek, H. E. : 1963, 'Magnetic Field Annihilation', in AAS-NASA Symp. on the Physics of Solar Flares, p. 425. Goddard Space Flight Center, Greenbelt, Maryland.

Spreiter, J. R., Summers, A. L., and Alksne, A. Y.: 1966, Planetary Space Sci. 14, 223. 